\title{
DEFORMATION-INDUCED SEGREGATION IN AUSTENITIC ALLOYS
}

\author{
S. A. Starikov, A. R. Kuznetsov*, Yu. N. Gornostyrev, V. V. Sagaradze \\ M.N. Miheev Institute of Metal Physics, Ural Branch of the Russian Academy of Sciences, \\ 18 S. Kovalevskoy St., Ekaterinburg, Russian Federation
}

\begin{abstract}
*Corresponding author. E-mail: a_kuznetsov@imp.uran.ru; address for correspondence: 18, ul. S. Kovalevskoy, 620990 Ekaterinburg, Russian Federation; phone: +7 (343) 37442 14; fax: +7 (343) 3745244
\end{abstract}

This article covers an overview of recent works devoted to the theoretical study of deformation-induced segregation in austenitic alloys as an example. A theoretical model describing nonequilibrium strain-induced segregation in a ternary alloy during severe plastic deformation is discussed. The model accounts for the generation of point defects, their annihilation at the sinks (such as grain boundaries), as well as mutual recombination. Based on the proposed model, the redistribution of the atoms of the alloying elements and the formation of grain boundary segregations during severe plastic deformation are investigated on the example of the $\mathrm{Fe}-12 \mathrm{Cr}-30 \mathrm{Ni}$ austenitic alloy. It is shown by the molecular dynamics method that in the Fe-30Ni binary alloy nickel atoms do not have their own thermodynamic incentives for segregation to the grain boundaries. The calculations demonstrate that the main contribution to the formation of segregations is due to the nonequilibrium flows of point defects (vacancies and internode atoms), which develop during severe plastic deformation. The obtained results explain the features of the formation of segregations in the $\mathrm{Fe}-\mathrm{Cr}-\mathrm{Ni}$ alloy during severe plastic deformation.

Keywords: severe plastic deformation, grain boundary, deformation-induced segregation, austenitic alloy.

DOI: $10.17804 / 2410-9908.2016 .6 .048-062$

\section{References}

1. Sagaradze V.V., Uvarov A.I. Uprochnenie $i$ svoistva austenitnykh stalei [Hardening and Properties of Austenitic Steels]. Ekaterinburg, UrO RAN Publ., 2013, 720 p. (In Russian).

2. Watanabe S., Takamatsu Y., Sakaguchi N., Takahashi H. Sink effect of grain boundary on radiation-induced segregation in austenitic stainless steel. Journal of Nuclear Materials, 2000, vols. 283-287, part 1, pp. 152-156. DOI: 10.1016/S0022-3115(00)00204-X.

3. Valiev R.Z., Zhilyaev A.P., Langdon T.G. Bulk nanostructured materials: Fundamentals and Applications, John Wiley \& Sons, Hoboken, NJ, USA, 2014, 436 p.

4. Deryagin A.I., Zavalishin V.A., Sagaradze V.V., Kuznetsov A.R. Low-temperature straininduced atomic segregation in chromium-nickel steels. The Physics of Metals and Metallography, 2000, vol. 89, no. 6, pp. 610-621.

5. Deryagin A.I., Zavalishin V.A., Sagaradze V.V., Kuznetsov A.R., Ivchenko V.A., Vildanova N.F., Efros B.M. Effect of composition and temperature on the redistribution of alloying elements in Fe-Cr-Ni alloys during cold deformation. The Physics of Metals and Metallography, 2008, vol. 106, no. 3, pp. 291-311. DOI: 10.1134/S0031918X08090093.

6. Ermakov A.E., Gapontsev V.L., Kondratiev V.V., Gornostyrev Yu.N. Deformation-induced phase instability in nanocrystalline alloys. Fizika Metallov i Metallovedenie, 1999, vol. 88, no. 3, pp. 5-12.

7. Gapontsev V.L., Deryagin A.I., Gapontseva T.M. Interpretation of temperature dependences of the composition distribution in nanostructured alloys under severe plastic deformation. Fizicheskaya mezomekhanika, 2009, vol. 12, no. 6, pp. 53-62. (In Russian).

8. $\quad$ Rozhanskiy V.N., Sizova N.L., Urusovskaya A.A. Crowdion plasticity CsI. Fizika tverdogo tela, 1971, vol. 13, iss. 2, pp. 411-415. (In Russian). 
9. Chaudhri M.N., Hagan J.T., Wells J.K. Observations of contact damage in MgO and LiF crystals by cathodoluminescence. Journal of Materials Science, 1980, vol. 15, no. 5, pp. 1189-1193.

10. Velednitskaya M.A., Rozhanskii V.N., Comolova L.F., Saparin G.V., Schreiber J., Brümmer O. Investigation of the deformation mechanism of $\mathrm{MgO}$ crystals affected by concentrated load. Physica Status Solidi (a), 1975, vol. 32, no. 1, pp. 123-132.

11. Golovin Yu.I., Tyurin A.I. Nondislocation plasticity and its role in the mass transfer and formation of the indentation under dynamic conditions. Physics of the Solid State, 2000, vol. 42, iss. 10, pp. 1865-1867. DOI: 10.1134/1.1318878.

12. Sosin A., Koehler J.S. Electrical resistivity tensor for aluminum single crystals deformed at helium temperature. Physical Review, 1956, vol. 101, no. 3, pp. 972-977.

13. Sosin A., Brinkman J.A. Electrical resistivity recovery in cold-worked and electronirradiated nickel. Acta Metallurgica, 1959, vol. 7, no. 7, pp. 478-494.

14. Meechan C.J., Sosin A. Recovery of electrical resistivity of $\mathrm{Cu}, \mathrm{Au}$, and $\mathrm{Ni}$ following cold work at $4^{\circ}$ K. Journal of Applied Physics, 1958, vol. 29, pp. 738-739.

15. Starikov S.A., Kuznetsov A.R., Karkina L.E., Sagaradze V.V. Ultimate theoretical strength of FCC Fe-Ni alloy polycrystals. Diagnostics, Resource and Mechanics of materials and structures, 2015, iss. 6, pp. 58-62. DOI: 10.17804/2410-9908.2015.6.058-062. Available at: http://dreamjournal.org/DREAM_Issue_6_2015_Starikov_S.A._et_al._058_062.pdf.

16. Kuznetsov A.R., Starikov S.A., Sagaradze V.V., Stepanov I.A., Pechenkin V.A., Giersig M. Studying deformation-induced segregation in the Fe-Cr-Ni alloy. The Physics of Metals and Metallography, 2004, vol. 98, no. 3, pp. 294-299.

17. Starikov S.A., Kuznetsov A.R., Sagaradze V.V., Pechenkin V.A., Stepanov I.A. The model of deformation-induced segregation near a moving grain boundary in the Fe-Cr-Ni alloy. The Physics of Metals and Metallography, 2006, vol. 102, no. 2, pp. 135-139. DOI: 10.1134/S0031918X06080035.

18. Starikov S.A., Kuznetsov A.R., Sagaradze V.V., Pechenkin V.A., Stepanov I.A. Influence of the temperature and rate of generation of point defects on the process of deformation-induced segregation in the Fe-Cr-Ni alloy. The Physics of Metals and Metallography, 2010, vol. 109, no. 4, pp. 376-382. DOI: 10.1134/S0031918X10040113.

19. Starikov S.A., Kuznetsov A.R., Sagaradze V.V., Gornostyrev Yu.N., Pechenkin V.A., Stepanov I.A. Formation of grain boundary segregations in alloy $\mathrm{Fe}-\mathrm{Cr}-\mathrm{Ni}$ during strong deformation and radiation. The Physics of Metals and Metallography, 2012, vol. 113, no. 3, pp. 241-245. DOI: 10.1134/S0031918X12030155.

20. XMD - Molecular Dynamics for Metals and Ceramics, computer software 2011. Available at: http://xmd.sourseforge.net/about.html. [18 February 2011].

21. Meyer R., Entel P. Martensite-austenite transition and phonon dispersion curves of $\mathrm{Fe}_{1-x} \mathrm{Ni}_{x}$ studied by molecular-dynamics simulations. Physical Review B, 1998, vol. 57, iss. 3, pp. 5140-5143. DOI: 10.1103/PhysRevB.57.5140.

22. Stepanov I.A., Pechenkin V.A. Kinetics of Radiation-Induced Segregation at Grain Boundaries in Fe-Cr-Ni Alloys. Metally, 2003, no. 6, pp. 84-90. (In Russian).

23. Marwick A.D. Calculation of bias due to solute redistribution in an irradiated binary alloy: surfaces of a thin foil. Journal of Nuclear Materials, 1985, vol. 135, iss. 1, pp. 68-76. DOI: 10.1016/0022-3115(85)90438-6.

24. Watanabe S., Sakaguchi N., Kurome K., Nakamura M., Takahashi H. On the mechanism of radiation-induced segregation. Journal of Nuclear Materials, 1997, vol. 240, iss. 3, pp. 251-253. DOI: 10.1016/S0022-3115(96)00710-6.

25. Lysova G.V., Birzhevoy G.A., Khramushin I.A. The investigation of the radiation-induced segregation of the elements near the surface of the Fe-20Cr-20Ni alloy after irradiation with iron ions. Journal of Surface Investigation: X-Ray, Synchrotron and Neutron Techniques, 2001, vol. 16, pp. 787-793. 
26. Sagaradze V.V., Shabashov V.A. Anomalous Diffusion Phase Transformations in Steels upon Severe Cold Deformation. The Physics of Metals and Metallography, 2011, vol. 112, no. 2, pp. 146-164. DOI: 10.1134/S0031918X11020256.

27. Starenchenko V.A., Pantyukhova O.D., Solov'eva Yu.V. Generation and accumulation of point defects in alloys with an L1(2) superstructure upon plastic deformation. The Physics of Metals and Metallography, 2004, vol. 97, no. 6, pp. 545-551.

28. Smirnov B.I. Vacancy Generation and Variation of Alkali Halide Crystal Density under Plastic Deformation. Fizika tverdogo tela, 1991, vol. 33, no. 9, pp. 2513-2526. (In Russian).

29. Kolupaeva S.N., Starenchenko V.A., Popov L.E. Neustoychivaya plasticheskaya deformatsiya kristallov [Instabilities of Plastic Deformation of Crystals]. Tomsk, Tomsk University Publ., 1994, 301 p. (In Russian).

30. Akhiezer I.A., Davydov L.N. Vvedenie v teoreticheskuyu radiatsionnuyu fiziku metallov $i$ splavov [Introduction into the Theoretical Radiation Physics of Metals and Alloys]. Kiev, Naukova Dumka Publ., 1985, 144 p. (In Russian).

31. Lejcek P. Grain Boundary Segregation in Metals, Springe, Heidelberg, Dordrecht, London, New York, 2010, 239 p.

32. Johnson R.A., Lam N.Q. Solute segregation in metals under irradiation. Physical Review B, 1976, vol. 13, iss 10, pp. 4364-4375. DOI: 10.1103/PhysRevB.13.4364.

33. Okamoto P.R., Rehn L.E. Radiation-induced segregation in binary and ternary alloys. Journal of Nuclear Materials, 1979, vol. 83, iss. 1, pp. 2-23. DOI: 10.1016/0022-3115(79)90587-7.

34. Stepanov I.A., Pechenkin V.A. Modeling of radiation-induced segregation at grain boundaries in Fe-Cr-Ni alloys. In: Trudy sedmoy Rossiysskoy konferentsii po reaktornomu materialovedeniyu. T. 3. Ch. 3 [Proceedings of the 7th Conference on Reactor Material Science, vol. 3, part 3]. Dimitrovgrad, NIIAR Publ., 2004, pp. 212-230. (In Russian). 
Подана в журнал: 11.11 .2016

УДК 669.1'26'24:620.186.2

DOI: $10.17804 / 2410-9908.2016 .6 .048-062$

\title{
ДЕФОРМАЦИОННО-ИНДУЦИРОВАННАЯ СЕГРЕГАЦИЯ В АУСТЕНИТНЫХ СПЛАВАХ
}

\author{
С. А. Стариков, А. Р. Кузнецов*, Ю. Н. Горностырев, В. В. Сагарадзе \\ Федеральное государственное бюджетное учреждение науки Институт физики металлов \\ имени М.Н. Михеева Уральского отделения Российской академии наук, ул. С.Ковалевской, 18, \\ 620990, Екатеринбург, Российская Федерация \\ *Ответственный автор. Электронная почта: a_kuznetsov@imp.uran.ru; \\ адрес для переписки: ул. С. Ковалевской, 18, 620990, Екатеринбург, Российская Федерация. \\ Телефон: +7 (343) 374-42-14; Факс: +7 (343) 374-52-44
}

Представлен обзор недавних работ, посвященных теоретическому исследованию деформационно-индуцированной сегрегации на примере аустенитных сплавов. Обсуждается теоретическая модель, описывающая неравновесную деформационно-индуцированную сегрегацию в трехкомпонентном сплаве при интенсивной пластической деформации. Модель учитывает рождение точечных дефектов в процессе интенсивной деформации, их аннигиляцию на стоках (таких как границы зерен), а также взаимную рекомбинацию. С использованием предложенной модели исследованы перераспределения атомов легирующих элементов и образования зернограничных сегрегаций при интенсивной пластической деформации на примере аустенитного сплава $\mathrm{Fe}-12 \mathrm{Cr}-30 \mathrm{Ni}$. Методом молекулярной динамики показано, что в бинарном сплаве $\mathrm{Fe}-30 \mathrm{Ni}$ атомы никеля не имеют собственных термодинамических стимулов для сегрегации на границы зерен. Расчеты показывают, что основной вклад в образование сегрегаций вносят неравновесные потоки точечных дефектов (вакансий и междоузельных атомов), формирующиеся при интенсивной пластической деформации. Полученные результаты позволяют объяснить особенности формирования сегрегаций при интенсивной пластической деформации сплава $\mathrm{Fe}-\mathrm{Cr}-\mathrm{Ni}$.

Ключевые слова: интенсивная пластическая деформация, граница зерна, деформаџионно-индуциированная сегрегация, аустенитный сплав.

\section{1. Ведение}

Интенсивная пластическая деформация (ИПД), как и радиационное облучение (РО), является одним из способов интенсивного внешнего воздействия на структуру и свойства сплавов. Во многих сплавах, подвергнутых как ИПД, так и РО, наблюдаются различные фазовые превращения, изменяющие физические и механические свойства этих материалов. При этом в поликристаллах существенную роль играет образование сегрегаций на границах зерен (ГЗ), особенно в случае ультрамелкозернистых материалов, структура которых формируется при ИПД $[1,2,3]$. Образующиеся при этом зернограничные сегрегации легирующих элементов могут существенно изменять их как механические, так и физические свойства. По этой причине исследование фазовых и структурных превращений при интенсивных внешних воздействиях является важным направлением физики материалов. Изучению этих явлений посвящены как экспериментальные $[4,5]$, так и теоретические работы $[6,7]$. Однако попытки теоретического описания наблюдаемого индуцированного расслоения твердого раствора были ограничены рассмотрением бинарного сплава и влияния на расслоение неравновесных вакансий без учета влияния междоузельных атомов, которые также генерируются при ИПД, о чем свидетельствуют экспериментальные работы [8-11], доказывающие междоузельную пластичность методом динамического индентирования. Помимо упомянутых выше экспери- 
ментов о генерации междоузлий говорят эксперименты, посвященные измерению электросопротивления деформированных образцов [12-14].

Таким образом, для более полного понимания механизмов, оказывающих влияние на изменения механических и физических свойств исследуемых сплавов при ИПД, необходимо более детальное изучение процессов деформационно-индуцированной сегрегации (ДИС) изменения распределения состава сплава при деформации вблизи стоков точечных дефектов.

\section{2. Постановка задачи и методы решения}

В настоящей статье рассматриваются как равновесная, так и неравновесная сегрегации, результаты исследований которых приведены соответственно в работах [15] и [16-19]. Для анализа равновесных сегрегаций [15] на ГЗ применен метод молекулярной динамики (МД) с использованием известного пакета программ XMD, разработанного в Центре компьютерного моделирования Университета Коннектикута (США) [20]. Использовались ЕАМ-потенциалы [21], хорошо апробированные для описания системы Fe-Ni. Моделирование проводилось для достаточно большого поликристалла, содержащего несколько зерен с границами наклона различного типа. В работе рассмотрена несимметричная специальная граница $\Sigma 5[001]\{710\} /\{110\}$ (ось разориентации [001], угол разориентации $\Theta=53,13^{\circ}$ ). На рис. 1 изображен тройной стык зерен, а также кристаллиты с изучаемой границей зерна $(\Sigma 5)$.

С целью определения термодинамических стимулов образования сегрегаций на ГЗ проведен расчет энергии взаимодействия атомов $\mathrm{Ni}$ с ГЗ в ГЦК-сплаве $\mathrm{Fe}-30 \mathrm{Ni}$. Поскольку в сплаве энергия легирующего элемента зависит от его локального окружения, энергия его взаимодействия с границей вычислялась путем усреднения по набору конфигураций (атом Ni замещал атом $\mathrm{Fe}$ на заданном расстоянии от ГЗ и считалась энергия кристаллита; такой расчет повторялся десятки раз для различных позиций атома легирующего элемента на заданном расстоянии от ГЗ, а затем полученная энергия усреднялась).

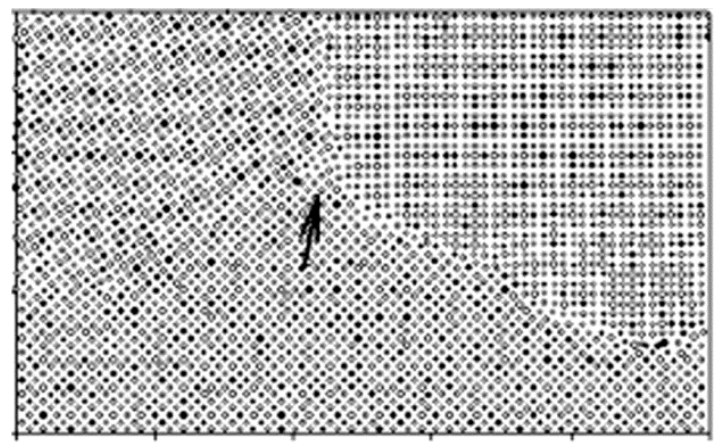

$a$

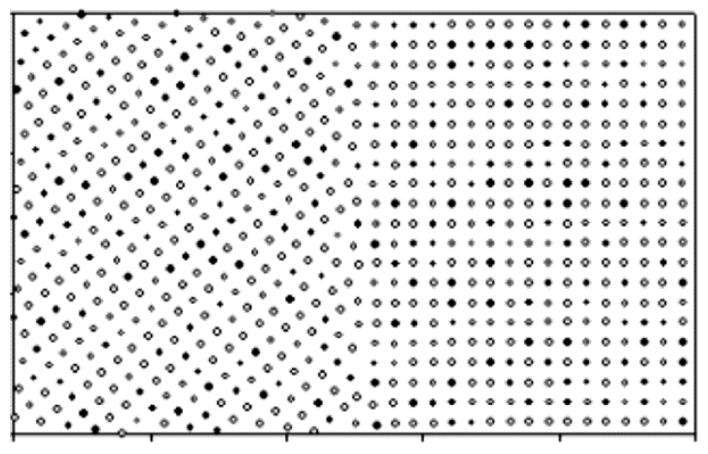

6

Рис. 1. Кристаллиты сплава Fe-30Ni (темные кружки - Ni) с тройным стыком $(a)$ (обозначен стрелкой) и с ГЗ $\Sigma 5[001]\{710\} /\{110\}$ (б) [15]

Для нахождения распределения компонент сплава, формирующегося под воздействием ИПД в модели неравновесной сегрегации [16] принято, как и в [4], что сегрегационные процессы, проходящие при деформации, аналогичны сегрегационным процессам, проходящим при облучении сплава. Отметим, что в настоящий момент нет разногласий касательно механизмов, вызывающих РИС. Однако, как отмечается в статье за 2003 год [22], до недавнего времени расчеты, связанные с РИС, проводили только с учетом вакансионного механизма РИС [23]. Однако тут же отмечается, что согласно последним исследованиям на примере сплава типа $\mathrm{Fe}-\mathrm{Cr}-\mathrm{Ni}$ были получены данные о РИС после низкотемпературного $\left(25-50{ }^{\circ} \mathrm{C}\right)$ электронного и ионного облучения [24, 25]. Поскольку при указанных выше температурах вакансии практически неподвижны, данные [24, 25] однозначно указывают на 
междоузельный механизм РИС. Таким образом, и при низкотемпературной деформации междоузельный механизм сегрегации также становиться доминирующим.

Образующиеся при деформации точечные дефекты (вакансии и междоузельные атомы) стекают на стоки, которыми являются в данном случае границы мелких зерен, а не движущиеся, образующиеся в процессе холодной деформации, дислокации, которые приводят к растворению сегрегаций, как это происходит с частицами интерметаллидов, растворяющимися в матрице аустенитных сплавов при взаимодействии с дислокациями при холодной деформации $[1,26]$.

Следуя результатам работы [27] мы полагаем, что при деформации генерируется сопоставимое количество вакансий и междоузельных атомов. Среди всех предложенных механизмов генерации точечных дефектов при деформации в настоящей работе принята точка зрения [28], согласно которой генерация точечных дефектов происходит главным образом за счет движения винтовых дислокаций со ступеньками атомных размеров. Образование этих ступенек осуществляется прежде всего путем двойного поперечного скольжения дислокаций, в результате которого происходит также размножение дислокаций и рождение дислокационных диполей. Как показано в [29] зависимость скорости изменения плотности винтовых дислокаций $d \rho / d t$ от температуры имеет вид

$$
d \rho / d t=-A_{1} \exp \left[-\frac{U}{k T}\right],
$$

где $A_{1}$ - коэффициент, зависящий в том числе от плотности дислокаций; $U$ - энергия активации; $k$ - постоянная Больцмана; $T$ - температура сплава.

При выводе формулы (1) было учтено, что: 1) преимущественно аннигилируют те винтовые дислокации, которые образуют устойчивый диполь по отношению к внешнему напряжению; 2) на одной из дислокаций диполя вследствие термической флуктуации происходит рекомбинация участка дислокации, в результате чего дислокация переходит в плоскость поперечного скольжения; 3) аннигилируют те диполи, расстояние между которыми таковы, что силы притяжения превышают силы трения, препятствующие встречному скольжению разноименных дислокаций.

Моделировался трехкомпонентный сплав $\mathrm{Fe}-\mathrm{Cr}-\mathrm{Ni}$, который подвергался большой пластической деформации на наковальнях Бриджмена. Считали, что атомы компонентов сплава до деформации равномерно распределены по всему объему и их начальные концентрации составляют $C_{a 0}=0,30, C_{b 0}=0,12, C_{c 0}=0,58$ (атомные доли), где индекс $a$ относится к никелю, $b$ - хрому, $c$ - железу.

В терминах парциальных коэффициентов диффузии выражения для потоков атомов и точечных дефектов (в системе координат, связанной с движущейся со скоростью $и$ границей зерна) имеют вид [22, 36, 17]

$$
\vec{j}_{\alpha}=-\left(d_{a v} C_{v}+d_{\alpha i} C_{i}\right) \vec{\nabla} C_{\alpha}+C_{\alpha}\left(d_{\alpha v} \vec{\nabla} C_{v}-d_{\alpha i} \vec{\nabla} C_{i}\right)-\vec{u} C_{\alpha}
$$

Для потока вакансий $\vec{j}_{v}$ и суммарного потока междоузельных атомов $\vec{j}_{i}$ в той же системе координат справедливы следующие соотношения:

$$
\begin{gathered}
\vec{j}_{v}=\left(d_{a v}-d_{c v}\right) C_{v} \vec{\nabla} C_{a}+\left(d_{b v}-d_{c v}\right) C_{v} \vec{\nabla} C_{b}-D_{v} \vec{\nabla} C_{v}-\vec{u} C_{v} \\
\vec{J}_{i}=\left(d_{a i}-d_{c i}\right) C_{i} \vec{\nabla} C_{a}+\left(d_{b i}-d_{c i}\right) C_{i} \vec{\nabla} C_{b}-D_{i} \vec{\nabla} C_{i}-\vec{u} C_{i} .
\end{gathered}
$$


Для нахождения распределений концентраций компонентов сплава и точечных дефектов использовалось гидродинамическое приближение, а именно уравнение непрерывности. При этом в уравнения для точечных дефектов (6) вводились дополнительные слагаемые, описывающие рождение и поглощение точечных дефектов, а также их взаимную рекомбинацию. Имеем

$$
\begin{gathered}
\partial C_{\alpha} / \partial t=-\vec{\nabla} \vec{j}_{\alpha} \\
\partial C_{n} / \partial t=-\vec{\nabla} \vec{j}_{n}+K_{g e n}^{n}-\mu_{R} D_{i} C_{i} C_{v}-k_{n s}^{2} D_{n}\left(C_{n}-C_{n 0}\right),
\end{gathered}
$$

где $n=i, v-$ индекс, обозначающий точечные дефекты (например, суммарную концентрацию междоузельных атомов $C_{i}$ и концентрацию вакансий $C_{v}$ соответственно); $\vec{j}_{\alpha}$ - вектор потока компонента $\alpha$ сплава; $\vec{j}_{n}$ - вектор потока точечных дефектов; $K_{g e n}^{n}-$ скорость генерации точечных дефектов типа $n$ при деформации; $\mu_{R}$ - постоянная рекомбинации; $k_{n s}^{2}-$ эффективность стоков точечных дефектов; $D_{n}=C_{a} d_{a n}+C_{b} d_{b n}+C_{c} d_{c n}$; здесь $d_{\alpha n}$ - парциальные коэффициенты диффузии $\alpha$-компоненты; $C_{\alpha}-$ концентрация $\alpha$-компоненты после деформации; $C_{n}$ - концентрация точечных дефектов сорта $n ; C_{n 0}$ - термически равновесная концентрация точечных дефектов сорта $n$, которая определяется следующим образом:

$$
C_{n 0}=\exp \left(S_{n}^{f} / k T\right) \times \exp \left(-E_{n}^{f} / k T\right)
$$

где $S_{n}^{f}$ и $E_{n}^{f}$ - энтропия и энергия образования, соответственно, точечных дефектов сорта $n$.

Учитывая, что при деформации генерируется сопоставимое количество вакансий и междоузельных атомов [27], о чем мы говорили выше, для скорости генерации точечных дефектов можно принять $K_{g e n}^{v}=K_{g e n}^{i}=K_{g e n}$.

Отметим, что второе, третье и четвертое слагаемые в правой части уравнения (6) описывают рождение, взаимную рекомбинацию и захват какими-либо поглотителями точечных дефектов соответственно. В уравнениях (3) и (4) первое и второе слагаемые описывают потоки Киркендалла вакансий и междоузельных атомов соответственно, возникающие в неоднородном по составу сплаве [30].

Систему диффузионных уравнений (4) и (5) необходимо дополнить начальными и граничными условиями [17]. Учитывая, что на границах зерен концентрация точечных дефектов равна термически равновесной, а в середине зерна потоки точечных дефектов равны нулю (в случае неподвижной границы зерна), можно записать:

$$
\begin{aligned}
& C_{n}(x, 0)=C_{n 0} ; \\
& C_{n}(0, t)=C_{n 0} ; \\
& J_{n}(L / 2, t)=0,
\end{aligned}
$$

где $L$ - размер зерна, принятый равным 100 нанометрам; $t$ - время деформации. Начальные и граничные условия для компонентов сплава имеют вид:

$$
C_{\alpha}(x, 0)=C_{\alpha 0}(x)
$$




$$
\begin{gathered}
J_{\alpha}(0, t)=0 ; \\
J_{\alpha}(L / 2, t)=0,
\end{gathered}
$$

где начальные концентрации $C_{\alpha 0}(x)$ - произвольные функции координат $\left(C_{a 0}(x)+C_{b 0}(x)+\right.$ $\left.+C_{c 0}(x)=1\right)$.

В случае движущейся границы зерна краевые условия записываются иначе за исключением условия (8). Предполагая концентрационную непрерывность компонентов сплава на границе зерна и считая, что граница зерна абсолютно прозрачна для потоков компонентов сплава, имеем:

$$
\begin{gathered}
C_{\alpha}(-0, t)=C_{\alpha}(+0, t) ; \\
J_{\alpha}(-0, t)=J_{\alpha}(+0, t) .
\end{gathered}
$$

Другим краевым условием служит периодичность концентраций компонентов сплава и точечных дефектов:

$$
\begin{aligned}
& C_{\alpha}(-L / 2, t)=C_{\alpha}(+L / 2, t) ; \\
& C_{n}(-L / 2, t)=C_{n}(+L / 2, t) .
\end{aligned}
$$

Система диффузионных уравнений (5) и (6) с краевыми условиями (7)-(16) решалась методом конечных разностей и аппроксимировалась интегро-интерполяционным методом на неравномерно пространственной сетке по чисто неявной схеме с помощью компьютерной программы RIS [22].

\section{3. Результаты и обсуждение}

На рис. 2 представлена полученная методом МД моделирования [16] зависимость разности средних энергий $E-E_{0}$ от расстояния $X$ до ГЗ (указаны доверительные интервалы), что соответствует средней энергии взаимодействия Ni с ГЗ; здесь $E$ - средняя по конфигурациям энергия кристаллита, в котором наблюдаемый атом $\mathrm{Ni}$ в сплаве $\mathrm{Fe}-30 \mathrm{Ni}$ находился на заданном расстоянии; $E_{0}$ - средняя по конфигурациям энергия кристаллита, в котором наблюдаемый атом Ni находился вдали от ГЗ. $X=0$ соответствует точке, расположенной в центре ГЗ.

Из рис. 2 следует, что атомы никеля довольно слабо взаимодействуют с данной специальной ГЗ (энергия сегрегации не превышает 0,05 эВ), поскольку имеют близкие к атомам матрицы ионный радиус и электронную структуру. По этой причине вклад равновесной сегрегации в общее обогащение ГЗ должен быть незначительным. В этом можно убедиться, проведя по полученным данным оценку равновесной сегрегации, используя individual site model [31]. Расчет показал, что при температуре $T=400^{\circ} \mathrm{K}$ концентрация никеля на ГЗ не превышает $35 \%$.

Таким образом, атомы никеля в сплаве $\mathrm{Fe}-30 \mathrm{Ni}$ не имеют собственных термодинамических стимулов для сегрегации на ГЗ. Это обстоятельство говорит о необходимости использовать теорию неравновесных сегрегаций для описания сегрегаций никеля на ГЗ в сплаве $\mathrm{Fe}-30$ ат. \% $\mathrm{Ni}$, используемом в МД-моделировании в качестве модельного для тройного сплава $\mathrm{Fe}-\mathrm{Cr}-\mathrm{Ni}$. 


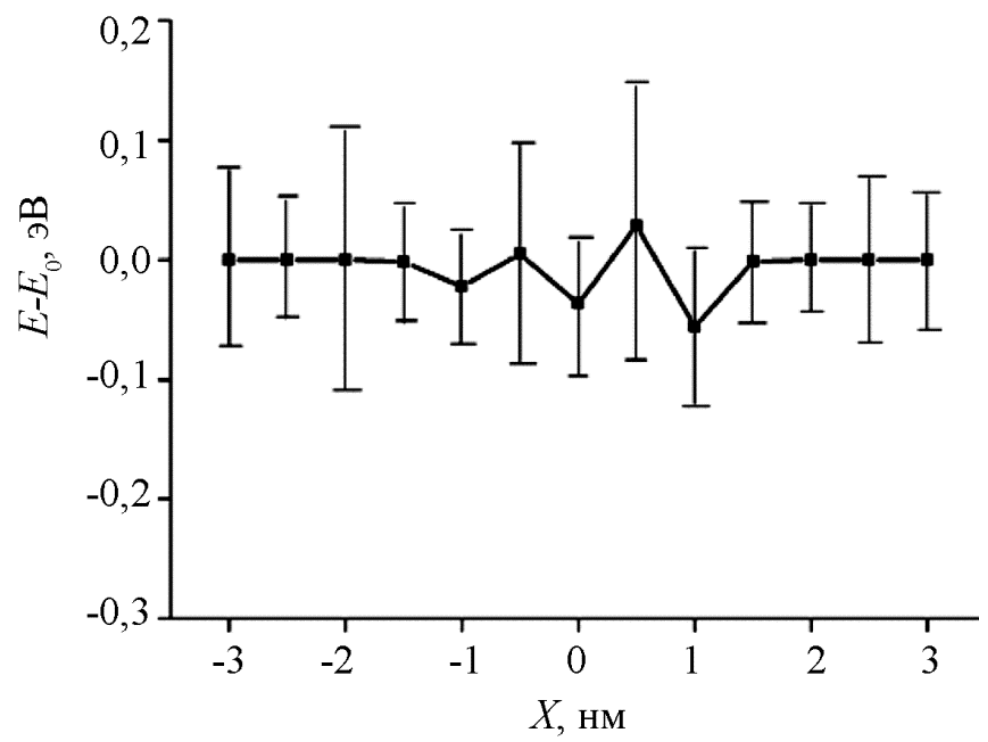

Рис. 2. Средняя энергия взаимодействия атомов Ni с ГЗ $\Sigma 5[001]\{710\} /\{110\}$

Профили концентраций компонентов сплава Х12Н30 в окрестности покоящейся ГЗ после деформации при $T=400^{\circ}$ К представлены на рис. 3 [16]. Время деформации составляло 600 с. Скорость генерации точечных дефектов $K_{\text {gen }}$ была оценена в [4] и составила значение, равное $10^{-4} \mathrm{c}^{-1}$. Видно, что в окрестности ГЗ происходит увеличение концентрации никеля по сравнению с начальным его значением за счет обеднения железом и хромом. Ширина обогащенной никелем зоны составляет несколько нанометров. В эту зону входит граница зерна и область сплава с существенно измененными концентрациями компонентов сплава по обе стороны от границы вплоть до значений концентраций, соответствующих значениям концентраций компонентов сплава до деформации. Данная зона влияет на изменение магнитных свойств изучаемого сплава.

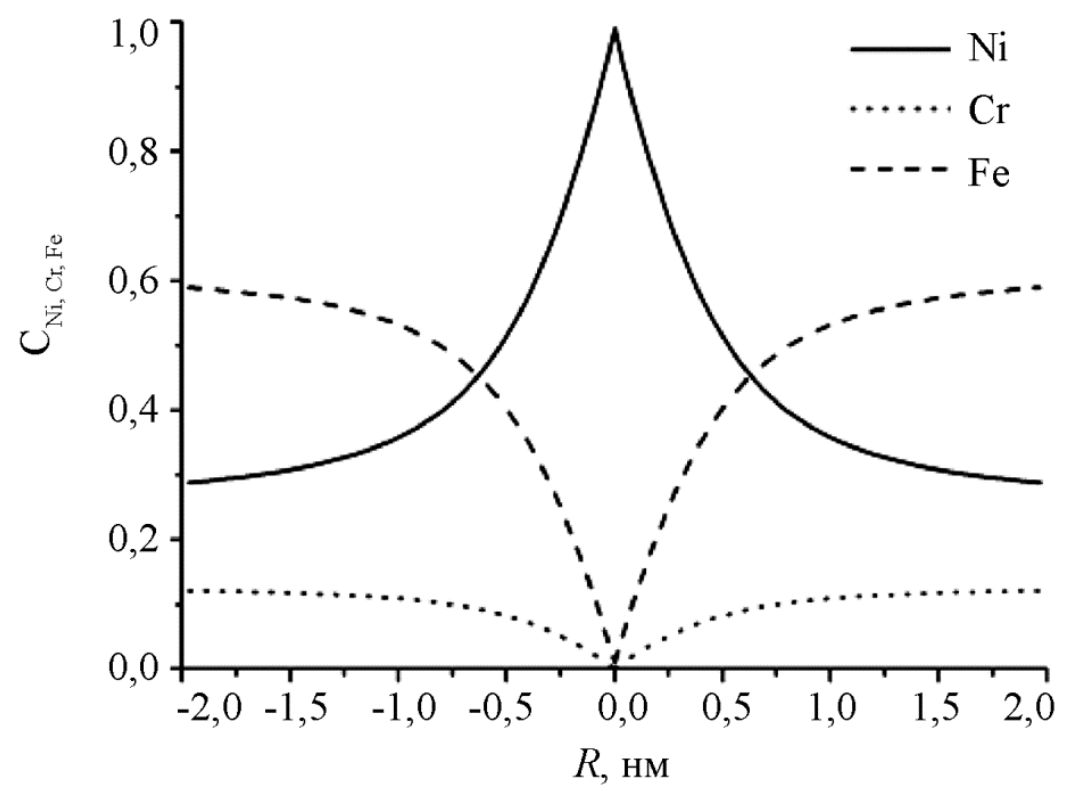

Рис. 3. Профили концентраций компонентов сплава Х12Н30 в окрестности покоящейся ГЗ (пояснения в тексте) [16]

Учет движения ГЗ [17] приводит к асимметрии в распределении вещества в приграничной зоне по сравнению с профилем концентрации, полученным для этого же сплава в случае покоящейся границы, которая представлена на рис. 4. Скорость движения ГЗ равна 0,003 нм/с и направлена слева направо. 


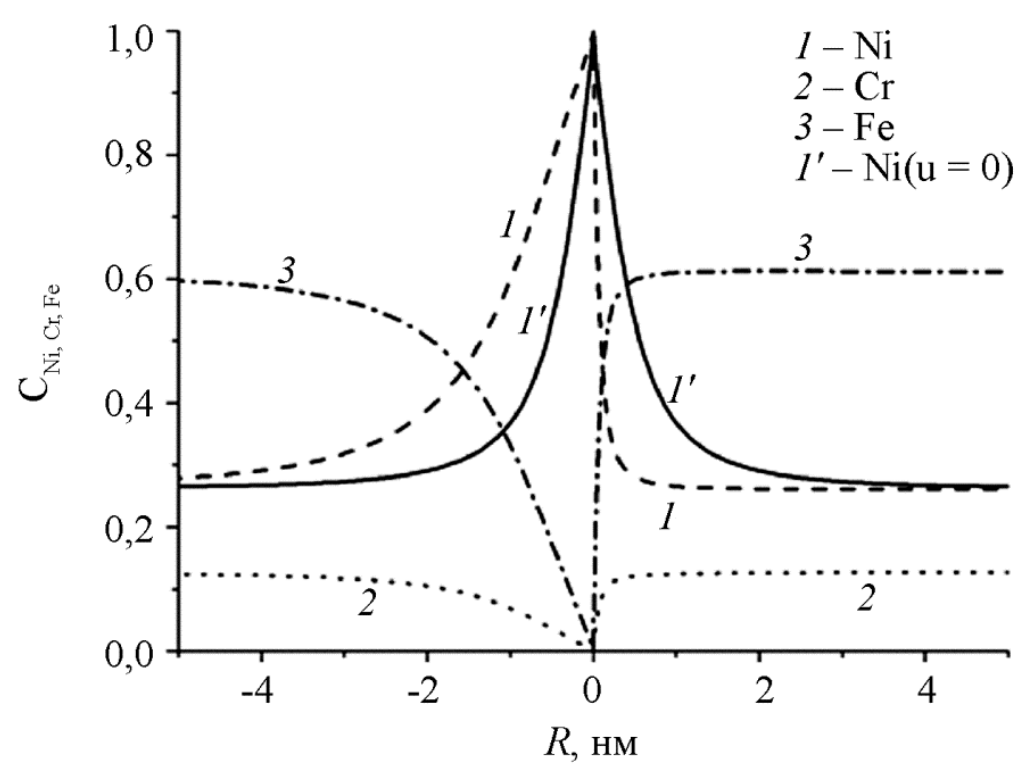

Рис. 4. Профили концентраций компонентов сплава Х12Н30 в случае движущейся Г3 [17]

На рис. 5 представлена зависимость усредненных значений концентраций компонентов сплава от температуры деформации в обогащенной никелем (и обедненной другими элементами) зоне, полученные путем усреднения в этой зоне после полного проведения деформации [16]. В качестве усредненного значения приближенно брали концентрацию на полувысоте пиков распределений $C(R)$, аналогичных приведенным на рис. 3. Иными словами, полувысота отсчитывалась от значения концентрации никеля до деформации. Эта зависимость здесь представлена в предположении, что скорость генерации точечных дефектов не зависит от температуры. Именно такая ситуация реализуется при рассмотрении радиационно-индуцированной сегрегации [22]. Вид полученных кривых качественно совпадает с аналогичными кривыми, которые приведены в работе для трехкомпонентного сплава [22]. Стоит отметить, что в пределе высоких температур $\left(T=950^{\circ} \mathrm{K}\right)$, концентрации компонентов стремятся к своему первоначальному значению. Иными словами, при высоких температурах деформация образца не приводит к пространственной неоднородности распределения компонентов сплава.

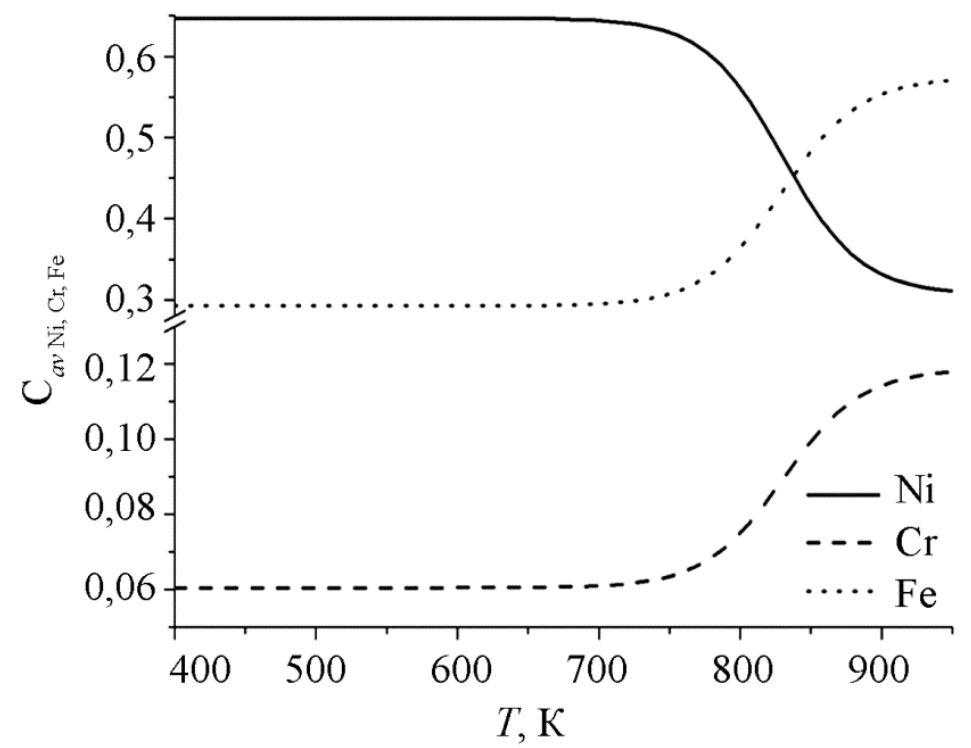

Рис. 5. Зависимость усредненных значений концентраций компонентов сплава Х12Н30 в случае покоящейся границы зерна от температуры деформации в предположении постоянства скорости генерации точечных дефектов во всем исследуемом температурном интервале, равной $10^{-4} \mathrm{c}^{-1}[16]$ 
Отметим, что учет движения ГЗ не приводит к качественному и количественному изменениям зависимости, представленной на рис. 5 для рассмотренного интервала скоростей.

Как показали эксперименты [5], увеличение температуры деформации действительно приводит к уменьшению сегрегационных процессов, проходящих в сплаве типа X11Н30. Однако полное исчезновение эффекта сегрегации происходит при более низких температуpax, чем это предсказывает зависимость на рис. 5. А именно при достижении температуры, равной $600^{\circ} \mathrm{K}$, концентрация никеля на границах зерен не отличается от его концентрации, которая была до деформации.

Для согласования теории и эксперимента, по нашему мнению, необходимо учесть тот факт, что скорость генерации точечных дефектов при деформации сильно понижается при повышении температуры вследствие протекания релаксационных процессов [28]. Это дает основание предложить [18], используя дополнительно соотношение (1), предполагая пропорциональную зависимость между скоростью генерации точечных дефектов и скоростью изменения плотности винтовых дислокаций, следующее выражение для скорости генерации точечных дефектов при деформации в зависимости от температуры

$$
K_{g e n}=K_{0}-K_{1} \exp \left[-\frac{U}{k T}\right],
$$

где $K_{0}$ - скорость генерации точечных дефектов при низких температурах, зависящая от скорости деформации. Отметим, что, хотя коэффициент $K_{1}$ и зависит от плотности дислокаций, в данной работе для упрощения он считался постоянным. При температурах $T>600 \mathrm{~K}$ и при $K_{1}>K_{0}$ из-за предположения о постоянстве коэффициента $K_{1}$ получаются отрицательные значения $K_{g e n}$; в этом случае мы принимаем $K_{g e n}=0$.

Были проанализированы [18] экспериментальные данные по скорости генерации точечных дефектов при деформации в зависимости от температуры и оказалось, что формула (17) хорошо описывает эксперимент.

На основе работы [5] для сплава Х12Н30 можно отметить следующие два значения на зависимости $K_{g e n}(T): K_{g e n} \approx 10^{-4} \mathrm{c}^{-1}$ при комнатной температуре и $K_{g e n} \approx 0$ при 600 К. Исходя из этого, были получены следующие значения параметров в выражении (3.1): $K_{0}=1,47 \cdot 10^{-4} \mathrm{c}^{-1}, K_{1}=3,38 \cdot 10^{-4} \mathrm{c}^{-1}, U=0,04$ эВ.

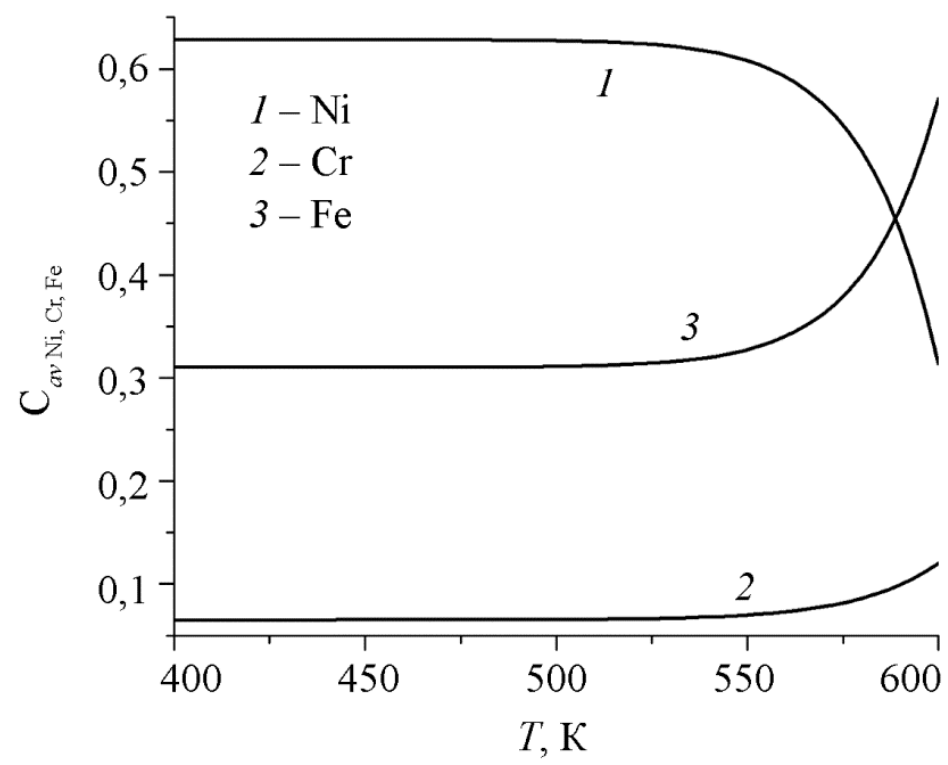

Рис. 6. Зависимости усредненных значений концентраций компонентов сплава Х12Н30 от температуры деформации, рассчитанные с учетом (17) [18] 
Используя выражение (17) для $K_{g e n}(T)$, мы провели расчет зависимостей усредненных значений концентраций компонентов $C_{a v}$, $C r$, Fe сплава X12H30 от температуры деформации [18]. Как видно из рис. 6, полученные результаты качественно согласуются с экспериментом [4] и сегрегация никеля на границу при температурах в районе температуры $600 \mathrm{~K}$ и более высоких не наблюдается.

На рис. 7 представлена зависимость [18] усредненных значений концентрации никеля $\frac{\Delta C_{a v}}{\Delta C_{a v \max }}=\frac{C_{\alpha v N i}-C_{a 0}}{C_{N i \max }^{a v}-C_{a 0}}$ (здесь $C_{N i \max }^{a v}-$ максимально возможное усредненное значение концентрации никеля), от скорости генерации точечных дефектов, когда постоянна суммарная концентрация сгенерированных точечных дефектов без учета их ухода на границу зерна, равная 0,06 (такой суммарной концентрации достаточно для того, чтобы проявился характер зависимости). На рис. 7 можно заметить, что увеличение скорости генерации точечных дефектов, начиная с некоторого его значения, приводит к уменьшению концентрации никеля на границе зерна (т.е. зависимость имеет максимум), качественно повторяя аналогичную зависимость при радиационно-индуцируемой сегрегации как от скорости генерации дефектов, так и от температуры [32]. Характерный вид зависимости на рис. 7 можно объяснить, приняв во внимание известный из исследований радиационно-индуцированной сегрегации факт, что в процессе увеличения скорости генерации точечных дефектов при фиксированной температуре увеличивается и скорость их рекомбинации $[32,33]$. Последнее заметно замедляет процесс сегрегации (в нашем случае - никеля) на границу зерна (следует также учитывать, что при повышении температуры возрастает обратная диффузия сегрегировавшего никеля от границы [33]).

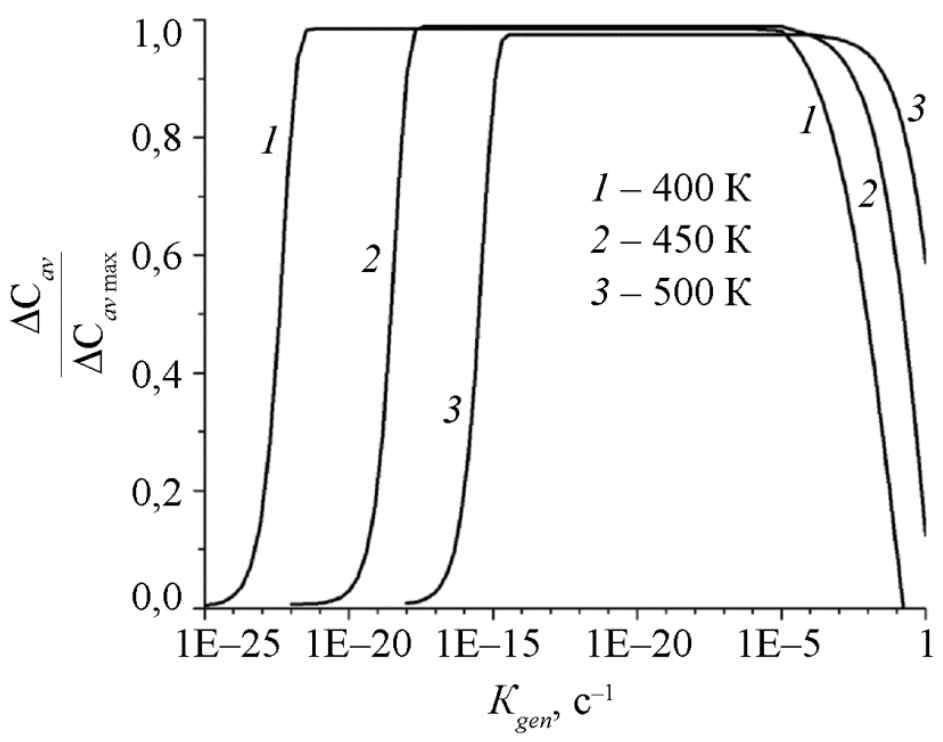

Рис. 7. Зависимость приведенных усредненных значений концентрации никеля $\frac{\Delta C_{a v}}{\Delta C_{a v \max }}$ от скорости генерации точечных дефектов при разных температурах деформации, когда постоянна суммарная концентрация сгенерированных точечных дефектов, равная 0,06 [18]

На рис. 8 представлена [19] зависимость усредненных значений концентрации никеля от времени деформации в обогащенной никелем зоне $C_{a v} \mathrm{Ni}$ покоящейся ГЗ при скорости генерации точечных дефектов $K_{g e n}=10^{-4} \mathrm{c}^{-1}$ и температуре деформации $400 \mathrm{~K}$, что соответствует экспериментальным условиям, реализующимся при сдвиге под давлением [4]. Сплав - Х12Н30. Из рис. 8 видно, что концентрация $C_{a v}$ м монотонно возрастает со временем деформации, достигая стационарного значения примерно за 10 минут. 
Символами на рис. 8 показаны усредненные значения концентрации никеля в обогащенной никелем зоне, полученные из эксперимента [4], соответствующие разным временам деформации. При выбранных значениях параметров расчетные кривые удовлетворительно согласуются с экспериментом. Монотонный характер кинетики ДИС сохраняется и при переходе к движущейся ГЗ (скорость движения принималась равной $0,003 \mathrm{Hм} / \mathrm{c}$ ). В то же время увеличение скорости генерации точечных дефектов до значения $10^{-2} \mathrm{c}^{-1}$ (такая величина $K_{g e n}$ реализуется, например, при холодной прокатке) приводит к немонотонной зависимости $C_{a v}{ }_{i}(t)$ (рис. 8, кривая 2) вследствие режима «быстрой» ДИС [34]; при этом максимальное значение $C_{a v}$ Ni достигается за короткие времена $\sim 10^{2}$ с.

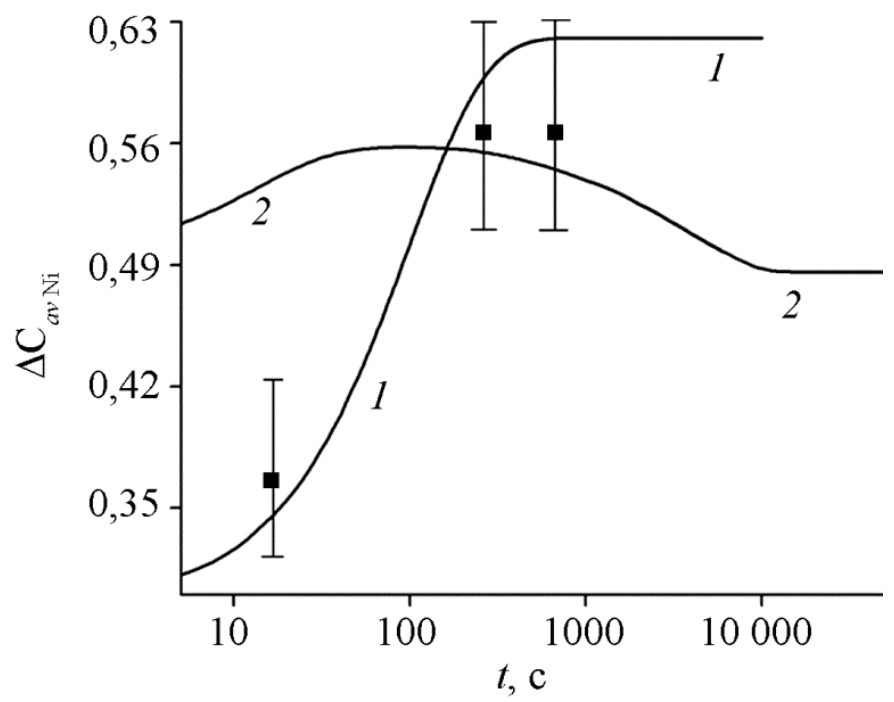

Рис. 8. Зависимости усредненных значений концентрации никеля от времени деформации [19]: $1-K_{\text {gen }}=10^{-4} \mathrm{c}^{-1}, T=400 \mathrm{~K}, u=0$; экспериментальные значения по данным [4] (匹); $2-K_{\text {gen }}=10^{-2} \mathrm{c}^{-1}, T=400 \mathrm{~K}, u=0,003 \mathrm{HM} / \mathrm{c}$

\section{4. Заключение}

Моделирование методом молекулярной динамики показало, что атомы никеля в аустенитном сплаве $\mathrm{Fe}-30 \mathrm{Ni}$ не имеют собственных термодинамических стимулов для сегрегации на ГЗ. Поэтому для описания сегрегаций никеля на ГЗ необходимо использовать теорию неравновесных сегрегаций. Предложенная модель неравновесной ДИС, учитывающая рождение и поглощение точечных дефектов, а также их взаимную рекомбинацию, распространена на случай трехкомпонентного сплава. Показана принципиальная возможность описания экспериментальных данных по ДИС с использованием разработанной теоретической модели. Выяснены основные механизмы неравновесной ДИС на примере тройного сплава $\mathrm{Fe}-\mathrm{Cr}-\mathrm{Ni}$ и проанализировано влияние основных параметров на ДИС. Показано, что кинетика ДИС может носить немонотонный характер.

\section{Благодарность}

Работа выполнена в рамках государственного задания ФАНО России по теме «Структура» № 01201463331. 


\section{Литература}

1. Сагарадзе В. В., Уваров А. И. Упрочнение и свойства аустенитных сталей. Екатеринбург: РИО УрО РАН, 2013. - 720 с.

2. Sink effect of grain boundary on radiation-induced segregation in austenitic stainless steel / S. Watanabe, Y. Takamatsu, N. Sakaguchi, H. Takahashi // Journal of Nuclear Materials. - 2000. Vols. 283-287, part 1. - P. 152-156. - DOI: 10.1016/S0022-3115(00)00204-X.

3. Valiev R. Z., Zhilyaev A. P., Langdon T. G. Bulk nanostructured materials: Fundamentals and Applications. - NJ: John Wiley and Sons, Hoboken, 2014. - 436 p.

4. Low-temperature strain-induced atomic segregation in chromium-nickel steels / A. I. Deryagin, V. A. Zavalishin, V. V. Sagaradze, A. R. Kuznetsov // The Physics of Metals and Metallography. - 2000. - Vol. 89, no. 6. - P. 610-621.

5. Effect of composition and temperature on the redistribution of alloying elements in $\mathrm{Fe}-\mathrm{Cr}-\mathrm{Ni}$ alloys during cold deformation / A. I. Deryagin, V. A. Zavalishin, V. V. Sagaradze, A. R. Kuznetsov, V. A. Ivchenko, N. F. Vildanova, B. M. Efros // The Physics of Metals and Metallography. 2008. - Vol. 106, no. 3. - P. 291-311. - DOI: 10.1134/S0031918X08090093.

6. Deformation-induced phase instability in nanocrystalline alloys / A. E. Ermakov, V. L. Gapontsev, V. V. Kondratiev, Yu. N. Gornostyrev // Fizika Metallov i Metallovedenie. 1999. - Vol. 88, no. 3. - P. 5-12.

7. Интерпретация зависимостей распределения состава наноструктурных сплавов, формируемых интенсивной пластической деформацией, от температуры деформации / В. Л. Гапонцев, А. И. Дерягин, Т. М. Гапонцева // Физическая мезомеханика. - 2009. - Т. 12, № 6. - С. 53-62.

8. Рожанский В. Н., Сизова Н. Л., Урусовская А. А. Краудионная пластичность CsI // Физика твердого тела. - 1971. - Т. 13, вып. 2. - С. 411-415.

9. Chaudhri M. N., Hagan J. T., Wells J. K. Observations of contact damage in $\mathrm{MgO}$ and $\mathrm{LiF}$ crystals by cathodoluminescence // Journal of Materials Science. - 1980. - Vol. 15, no. 5. P. 1189-1193.

10. Investigation of the deformation mechanism of $\mathrm{MgO}$ crystals affected by concentrated load / M. A. Velednitskaya, V. N. Rozhanskii, L. F. Comolova, G. V. Saparin, J. Schreiber, O. Brümmer // Physica Status Solidi (a). - 1975. - Vol. 32, no. 1. - P. 123-132.

11. Golovin Yu. I., Tyurin A. I. Nondislocation plasticity and its role in the mass transfer and formation of the indentation under dynamic conditions // Physics of the Solid State. - 2000. Vol. 42, iss. 10. - P. 1865-1867. - DOI: 10.1134/1.1318878.

12. Sosin A., Koehler J. S. Electrical resistivity tensor for aluminum single crystals deformed at helium temperature // Physical Review. - 1956. - Vol. 101, no. 3. - P. 972-977.

13. Sosin A., Brinkman J. A. Electrical resistivity recovery in cold-worked and electronirradiated nickel // Acta Metallurgica. - 1959. - Vol. 7, no. 7. - P. 478-494.

14. Meechan C. J., Sosin A. Recovery of electrical resistivity of $\mathrm{Cu}, \mathrm{Au}$, and $\mathrm{Ni}$ following cold work at $4^{\circ} \mathrm{K} / /$ Journal of Applied Physics. - 1958. - Vol. 29. - P. 738-739.

15. Ultimate theoretical strength of FCC Fe-Ni alloy polycrystals / S. A. Starikov, A. R. Kuznetsov, L. E. Karkina, V. V. Sagaradze // Diagnostics, Resource and Mechanics of materials and structures. - 2015. - Iss. 6. - P. 58-62. - DOI: 10.17804/2410-9908.2015.6.058-062. URL: http://dream-journal.org/DREAM_Issue_6_2015_Starikov_S.A._et_al._058_062.pdf.

16. Studying deformation-induced segregation in the $\mathrm{Fe}-\mathrm{Cr}-\mathrm{Ni}$ alloy / A. R. Kuznetsov, S. A. Starikov, V. V. Sagaradze, I. A. Stepanov, V. A. Pechenkin, M. Giersig // The Physics of Metals and Metallography. - 2004. - Vol. 98, no. 3. - P. 294-299.

17. The model of deformation-induced segregation near a moving grain boundary in the $\mathrm{Fe}-\mathrm{Cr}-\mathrm{Ni}$ alloy / S. A. Starikov, A. R. Kuznetsov, V. V. Sagaradze, V. A. Pechenkin, I. A. Stepanov // The Physics of Metals and Metallography. - 2006. - Vol. 102, no. 2. - P. 135-139. DOI: $10.1134 / \mathrm{S} 0031918 X 06080035$. 
18. Influence of the temperature and rate of generation of point defects on the process of deformation-induced segregation in the $\mathrm{Fe}-\mathrm{Cr}-\mathrm{Ni}$ alloy / S. A. Starikov, A. R. Kuznetsov, V. V. Sagaradze, V. A. Pechenkin, I. A. Stepanov // The Physics of Metals and Metallography. 2010. - Vol. 109, no. 4. - P. 376-382. - DOI: 10.1134/S0031918X10040113.

19. Formation of grain boundary segregations in alloy $\mathrm{Fe}-\mathrm{Cr}-\mathrm{Ni}$ during strong deformation and radiation / S. A. Starikov, A. R. Kuznetsov, V. V. Sagaradze, Yu. N. Gornostyrev, V. A. Pechenkin, I. A. Stepanov // The Physics of Metals and Metallography. - 2012. - Vol. 113, no. 3. - P. 241-245. DOI: 10.1134/S0031918X12030155.

20. XMD - Molecular Dynamics for Metals and Ceramics, computer software. - 2011. - URL: http://xmd.sourseforge.net/about.html.

21. Meyer R., Entel P. Martensite-austenite transition and phonon dispersion curves of $\mathrm{Fe}_{1-x} \mathrm{Ni}_{x}$ studied by molecular-dynamics simulations // Physical Review B. - 1998. - Vol. 57, iss. 3. P. 5140-5143. - DOI: 10.1103/PhysRevB.57.5140.

22. Степанов И. А., Печенкин В. А. Кинетика радиационно-индуцированной сегрегации на границах зерен в сплавах $\mathrm{Fe}-\mathrm{Cr}-\mathrm{Ni} / /$ Металлы. - 2003. - № 6. - С. 84-90.

23. Marwick A. D. Calculation of bias due to solute redistribution in an irradiated binary alloy: surfaces of a thin foil // Journal of Nuclear Materials. - 1985. - Vol. 135, iss. 1. - P. 68-76. DOI: 10.1016/0022-3115(85)90438-6.

24. Watanabe S., Sakaguchi N., Kurome K. On the mechanism of radiation-induced segregation // Journal of Nuclear Materials. - 1997. - Vol. 240, iss. 3. - P. 251-253. - DOI: 10.1016/S00223115(96)00710-6.

25. Lysova G. V. Birzhevoy G. A., Khramushin I. A. The investigation of the radiation-induced segregation of the elements near the surface of the Fe-20Cr-20Ni alloy after irradiation with iron ions // Journal of Surface Investigation: X-Ray, Synchrotron and Neutron Techniques. - 2001. Vol. 16. - P. 787-793.

26. Sagaradze V. V., Shabashov V. A. Anomalous Diffusion Phase Transformations in Steels upon Severe Cold Deformation // The Physics of Metals and Metallography. - 2011. - Vol. 112, no. 2. - P. 146-164. - DOI: 10.1134/S0031918X11020256.

27. Starenchenko V. A., Pantyukhova O. D., Solov'eva Yu. V. Generation and accumulation of point defects in alloys with an L1(2) superstructure upon plastic deformation // The Physics of Metals and Metallography. - 2004. - Vol. 97, no. 6. - P. 545-551.

28. Smirnov B. I. Vacancy Generation and Variation of Alkali Halide Crystal Density under Plastic Deformation // Fizika tverdogo tela. - 1991. - Vol. 33, no. 9. - P. 2513-2526.

29. Колупаева С. Н., Старенченко В. А., Попов Л. Е. Неустойчивая пластическая деформация кристаллов. - Томск: Изд-во Томского университета, 1994. - $301 \mathrm{c.}$

30. Ахиезер И. А., Давыдов Л. Н. Введение в теоретическую радиационную физику металлов и сплавов. - Киев : Наукова думка, 1985. - 144 с.

31. Lejcek P. Grain boundary segregation in metals. - Heidelberg, Dordrecht, London, New York: Springer, 2010. - 239 p.

32. Johnson R. A., Lam N. Q. Solute segregation in metals under irradiation // Physical Review B. - 1976. - Vol. 13, iss 10. - P. 4364-4375. - DOI: 10.1103/PhysRevB.13.4364.

33. Okamoto P. R., Rehn L. E. Radiation-induced segregation in binary and ternary alloys // Journal of Nuclear Materials. - 1979. - Vol. 83, iss. 1. - P. 2-23. - DOI: 10.1016/00223115(79)90587-7.

34. Степанов И. А., Печёнкин В. А. Моделирование радиационно-индуцированной сегрегации на границах зерен в $\mathrm{Fe}-\mathrm{Cr}-\mathrm{Ni}$ сплавах // Труды седьмой Российской конференции по реакторному материаловедению. - Димитровград : НИИАР, 2004. - Т. 3, ч. 3. - С. 212-230. 